\title{
High-resolution near-infrared study of the deeply embedded young stellar object S140 IRS 3*
}

\author{
T. Preibisch ${ }^{1}$, Y. Y. Balega ${ }^{2}$, D. Schertl ${ }^{1}$, M. D. Smith ${ }^{3}$, and G. Weigelt ${ }^{1}$ \\ 1 Max-Planck-Institut für Radioastronomie, Auf dem Hügel 69, 53121 Bonn, Germany \\ 2 Special Astrophysical Observatory, Nizhnij Arkhyz, Zelenchuk region, Karachai-Cherkesia 357147, Russia \\ 3 Armagh Observatory, College Hill, Armagh BT61 9DG, Northern Ireland
}

Received 9 May 2001 / Accepted 5 June 2001

\begin{abstract}
We explore the structures immediately surrounding the high-mass young stellar object S140 IRS 3 within the L1204 molecular cloud. We have obtained a bispectrum speckle interferometric $K$-band image with a resolution of 150 mas and a seeing-limited molecular hydrogen line emission image of IRS 3. Our speckle image resolves IRS 3 into three point sources, a close binary with separation $0.63^{\prime \prime}$ and a third component $1.3^{\prime \prime}$ away. A rough assessment of the system stability suggests that the IRS 3 triple system is unstable. Our speckle image also reveals extended diffuse emission of very complex morphology around IRS 3. An extended diffuse feature north-east of IRS 3 displays a remarkable S-shaped structure. This feature is the innermost part of an at least $15^{\prime \prime}$ long extended structure, which is pointing towards a bow-shock like patch located $90^{\prime \prime}$ away from IRS 3 . We find strong $\mathrm{H}_{2}$ line emission associated with this feature, suggesting the presence of shocks, caused by the collision of outflowing material with the ambient medium. The S-shaped structure of this feature can be well reproduced by a model assuming a precessing outflow from IRS 3a. Furthermore, we find several elongated features pointing away from IRS 3 in a southern direction. Some of these features also exhibit strong $\mathrm{H}_{2}$ line emission, demonstrating that IRS 3 drives outflows in several directions.
\end{abstract}

Key words. techniques: interferometric - stars: individual: S140 IRS3 - stars: formation - stars: winds, outflows

\section{Introduction}

S140 is a HII region at the south-east edge of the L1204 dark cloud, located at a distance of $\sim 900$ pc (Crampton \& Fisher 1974). This cloud contains a small cluster of highly obscured, optically invisible infrared sources, originally detected by Rouan et al. (1977). The $20 \mu \mathrm{m}$ observations by Beichman et al. (1979) revealed three individual infrared sources in this region. The brightest source is called IRS 1 , the two other sources IRS 2 and IRS 3 are located $\sim 17^{\prime \prime}$ north and $\sim 9^{\prime \prime}$ east of IRS 1 . The infrared spectra of these sources rise steeply between $10 \mu \mathrm{m}$ and $50 \mu \mathrm{m}$ (Lester et al. 1986), demonstrating that they are deeply embedded young stellar objects associated with

Send offprint requests to: T. Preibisch, e-mail: preib@mpifr-bonn.mpg.de

* Part of the results presented in this paper are based on observations obtained at the German-Spanish Astronomical Centre, Calar Alto, operated by the Max-Planck-Institute for Astronomy, Heidelberg, jointly with the Spanish National Commission for Astronomy. The speckle observations were collected at the Special Astrophysical Observatory with the $6 \mathrm{~m}$ telecope. circumstellar material. The luminosity of IRS 3 was estimated at $L \approx 3 \times 10^{3} L_{\odot}$ (Lester et al. 1986), suggesting it to be an early B-type star with a mass of roughly $8 M_{\odot}$.

S140 IRS is a well known source of strong molecular outflows. A bipolar $\mathrm{CO}$ outflow was first detected by Blair et al. (1978) and studied in more detail by Minchin et al. (1993). S140 IRS 1 lies just in the middle between the blue- and red-shifted outflow lobes. Since the other two infrared sources IRS 2 and IRS 3 are clearly not located on the axis of the outflow, IRS 1 can be assumed to be the source of this outflow. Further molecular line emission maps of the region were presented for example by Minchin et al. (1993; CO), Hayashi \& Murata (1992; CO), and Wilner \& Welch (1994; $\mathrm{HCO}^{+}$and SO). While the morphology of the molecular line emission in all these observations is consistent with the idea that IRS 1 is the driving source of the outflows, the spatial resolution of the maps is far too coarse to exclude the possibility that IRS 2 or IRS 3 also contribute to the outflow activity. What seems to be clear, however, is that IRS 1 dominates the outflow activity.

In previous studies, near-infrared images of the S140 IRS region were obtained for example by Lenzen (1987), 
Harker et al. (1997), Whitney et al. (1997), and Yao et al. (1998). These observations, however, were seeinglimited and therefore did not have the resolution required to study the environment ( $\$ 1000$ AU) of the individual sources in S140 IRS. This paper represents the third step in our high-resolution study of the S140 star-forming region. In the first paper (Schertl et al. 2000) we presented a 76 mas resolution bispectrum speckle interferometric $K$-band image of the central $3^{\prime \prime} \times 3^{\prime \prime}$ region around IRS 1 , and also polarimetric measurements. This image showed a bright elongated clumpy structure pointing away from IRS 1 in a direction consistent with that of the blue-shifted $\mathrm{CO}$ outflow lobe. The centrosymmetric pattern of high polarization in this feature suggests that it represents scattered light from the central source IRS 1. We therefore interpreted this feature as the clumpy inner edge of a partially evacuated cavity in the circumstellar envelope around IRS1, which has been excavated by the southeastern wing of the strong outflow from IRS 1 . In the second paper (Weigelt et al. 2001) we presented bispectrum speckle interferometry observations of a larger $13^{\prime \prime} \times 21^{\prime \prime}$ field of view of S140 IRS 1 and its environment. In addition to four new point sources, we found several arc-like structures around IRS 1. The general appearance of the diffuse structures suggests that they trace the interaction of the energetic outflows from IRS 1 with the circumstellar material. In combination with molecular line emission maps from the literature, our image provided evidence for the presence of flows in four different directions, all apparently originating from IRS 1.

The wealth of interesting structures revealed by our images of S140 IRS 1 was the motivation for extending our high-resolution imaging study of the S140 region to IRS 3. Here we present the results of our bispectrum speckle interferometry observations of IRS 3 in the $K$-band. We also obtained images in the light of the molecular hydrogen emission line at $2.12 \mu \mathrm{m}$. Molecular hydrogen emission is a convenient tracer of low- to moderate-velocity shocks (e.g. Smith 1993) and will give us important information about the outflow activity of the young stellar objects.

\section{Observations and data reduction}

\subsection{Bispectrum speckle imaging}

The speckle interferograms were recorded with the $6 \mathrm{~m}$ SAO telescope on 7, 14 and 15 October 2000. The detector of our speckle camera was a Rockwell HAWAII array detector. The observations were made through a $K$-band filter with center wavelength/ FWHM bandwidth of $2.12 \mu \mathrm{m} / 0.21 \mu \mathrm{m}$. The pixel size was 27.0 mas, the exposure time per frame was $240 \mathrm{~ms}$. 2216 speckle interferograms of S140 IRS 3 and 5826 speckle interferograms of the reference star HIP 110410 were obtained. The corresponding effective telescope focal length was obtained by using $K$-band re-imaging optics designed for diffractionlimited speckle imaging with the SAO telescope.
The field-of-view was $10.8^{\prime \prime} \times 10.8^{\prime \prime}(400 \times 400$ pixel $)$, seeing was $\sim 1^{\prime \prime}(F W H M)$.

Diffraction-limited images were reconstructed using the bispectrum speckle interferometry method (Weigelt 1977; Lohmann et al. 1983; Hofmann \& Weigelt 1986). The bispectrum of each frame consisted of 12 million elements. The object power spectrum was determined with the speckle interferometry method (Labeyrie 1970). Speckle interferograms of unresolved single stars were recorded just before and after the object, and served as reference stars for the determination of the speckle transfer function. The resulting $K$-band image has a resolution of 150 mas.

\subsection{Molecular hydrogen images}

Conventional near-infrared images of the S140 IRS region were obtained in October 2000 with the Omega Prime near-IR camera (Bizenberger et al. 1998) on the Calar Alto $3.5 \mathrm{~m}$ telescope. The pixel scale of Omega Prime is $0.4^{\prime \prime}$ per pixel, seeing was $\sim 1.4^{\prime \prime}$. Images were taken through the NB2122 filter, a $1 \%$ filter centered on the $v=1-0 S(1)$ line of the $\mathrm{H}_{2}$ molecule at $2.12 \mu \mathrm{m}$, and through a broad-band $K^{\prime}$ filter $(1.944-2.292 \mu \mathrm{m})$. At each of 6 different dither positions, a series of 10 images with 10 s exposure time in the NB2122 filter and of 10 images with $1.677 \mathrm{~s}$ exposure time in the $K^{\prime}$ filter were taken. The total net integration time is therefore $10 \mathrm{~min}$ in the NB2122 filter and $1.677 \mathrm{~min}$ in the $K^{\prime}$ filter. Standard data reduction techniques were used to sky subtract, flat field, and mosaic the data (cf. McCaughrean et al. 1994). Then, the NB2122 filter image was carefully aligned with the $K^{\prime}$ filter image via several point sources in the outer parts of the image. Finally, the $K^{\prime}$ filter image was subtracted from the NB2122 filter image in order to create the continuumsubtracted $\mathrm{H}_{2}$ emission line image.

\section{Results}

In Fig. 1 we present our images of S140 IRS 3. The most important features revealed by our speckle image are as follows:

- IRS 3 is clearly resolved into three point sources. The angular separation of the two brightest components, which we will call IRS $3 \mathrm{a}$ and IRS $3 \mathrm{~b}$, is $(627 \pm 10)$ mas with a position angle of $85^{\circ} \pm 1^{\circ}$. IRS $3 \mathrm{~b}$ is $(1.2 \pm 0.2)$ mag fainter than IRS $3 \mathrm{a}$ in the $K$-band. The third component, IRS 3c, is located $(1306 \pm 30)$ mas south of IRS 3a at a position angle of $172^{\circ} \pm 2^{\circ}$. IRS $3 \mathrm{c}$ is $(2.5 \pm 0.4)$ mag fainter than IRS 3a;

- several bright and extended features of diffuse emission are pointing from IRS 3a towards the south-east. These features are marked with the letters $\mathbf{A}, \mathbf{B}$, and $\mathbf{C}$ in Fig. 1. The direction of the innermost and brightest feature $\mathbf{A}$ has a position angle of $\sim 170^{\circ}$. Feature $\mathbf{B}$ has a position angle of $\sim 120^{\circ}$. Feature $\mathbf{C}$ is a strongly curved arc of diffuse emission south of IRS 3a; 

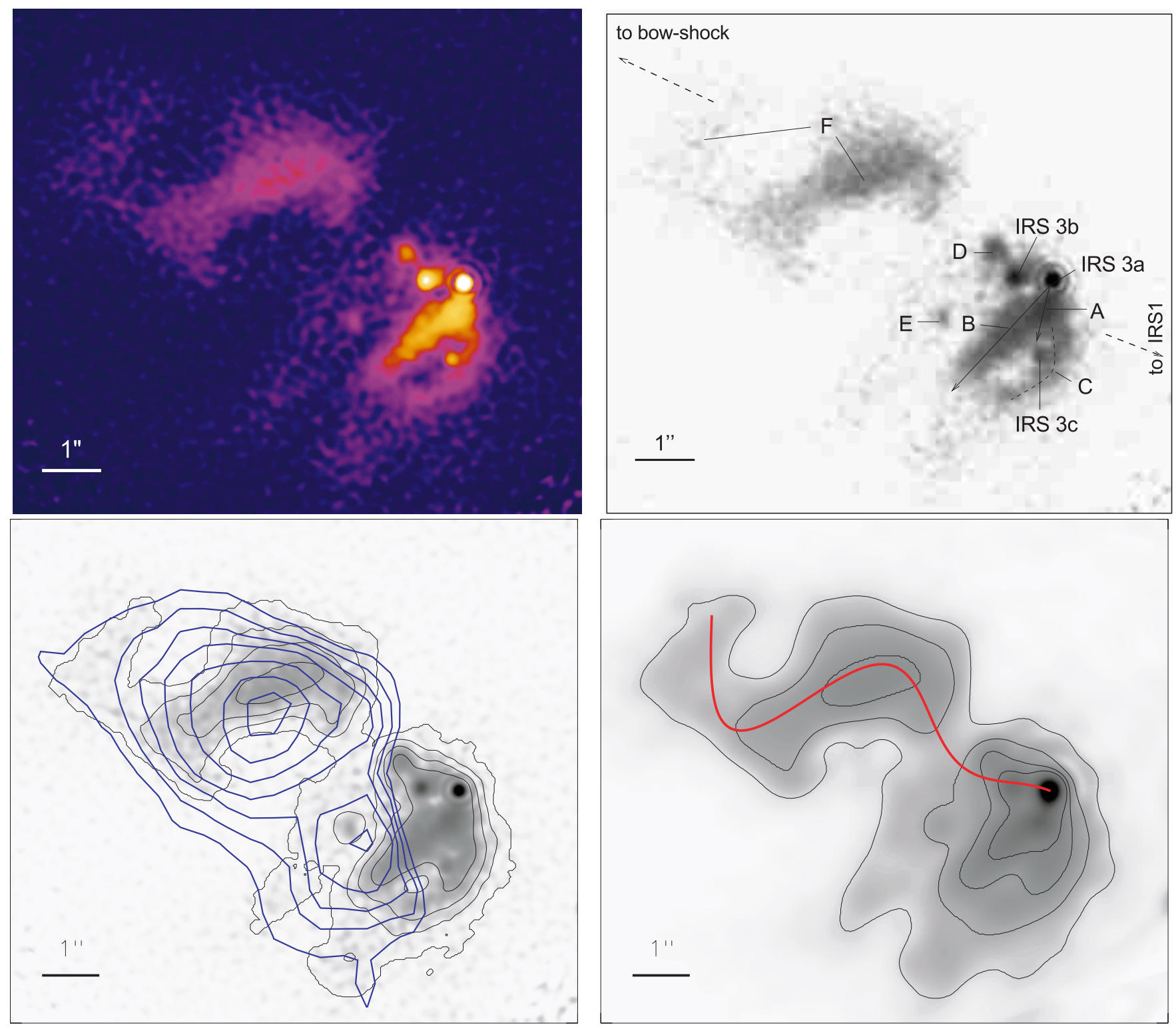

Fig. 1. Images of S140 IRS 3. In all images north is up and east is to the left; the field of view is $9.6^{\prime \prime} \times 8.5^{\prime \prime}$. Upper Left: Color representation of our 150 mas resolution bispectrum speckle $K$-band image of S140 IRS 3. Blue areas are $\sim 4$ mag fainter than the peak intensity. Upper Right: Greyscale representation of the $K$-band speckle image with annotation of the features discussed in the text. The dashed-lined arrow near the right edge of the image indicates the direction towards S140 IRS 1. Lower Left: Greyscale representation of the $K$-band speckle image; the black contour lines show a smoothed representation of this image in order to pronounce the structure of the faintest diffuse emission features. The thick blue contours show the continuum-subtracted $\mathrm{H}_{2}$ emission line image. Contours are drawn at 8, 10,12, 14, 17, 20, and 22 times the rms noise level above the median in the continuum-subtracted image. The estimated accuracy of the alignment between the $\mathrm{H}_{2}$ image and the speckle image is $\sim 0.2^{\prime \prime}$. Lower Right: Greyscale + contour representation of our $K$-band speckle image reconstructed with a reduced resolution of 420 mas in order to better show the fainter details. The thick red line shows the model curve computed from the precessing jet model (Eq. (1)).

- a patch of apparently diffuse emission (D) is found $\sim 0.5^{\prime \prime}$ north-east of IRS 3b, another one $(\mathbf{E})$ is located $\sim 1^{\prime \prime}$ south-east of IRS $3 \mathrm{~b}$;

- extended diffuse emission $(\mathbf{F})$ is found $\sim 2^{\prime \prime}$ north-east of IRS $3 \mathrm{~b}$. This feature displays a remarkable S-shaped structure (see Fig. 1, lower-right).
Comparison of the speckle image with our $\mathrm{H}_{2}$ emission line image (Fig. 1 lower left) yields the following results:

- Strong extended $\mathrm{H}_{2}$ emission is found in the northeastern part of our image, coinciding with feature $\mathbf{F}$ in the speckle image. 
- Another peak of $\mathrm{H}_{2}$ emission is found south-east of IRS 3 b. It coincides roughly with feature $\mathbf{B}$ and extends to the southern tip of feature $\mathbf{C}$.

\section{Interpretation}

\subsection{Feature $\mathbf{F}$}

\subsubsection{Scattered light and shock emission}

Feature $\mathbf{F}$ does not only show strong $\mathrm{H}_{2}$ line emission, but is also strongly polarized. The polarization pattern of feature $\mathbf{F}$ in the $K$-band polarimetric images of the S 140 region presented by Whitney et al. (1997) and Yao et al. (1998) suggests that we see scattered light originating from IRS 3. Comparison of the $\mathrm{H}_{2}$ and $K^{\prime}$ fluxes integrated over an 2.5" aperture centered on the $\mathrm{H}_{2}$ emission peak shows that $\sim 20 \%$ of the flux is $\mathrm{H}_{2}$ line emission, while $\sim 80 \%$ of the flux is continuum emission. Feature $\mathbf{F}$ probably represents a denser region in the environment of IRS 3, where light from IRS 3 is reflected in our direction, causing the observed high degree of polarization, and where outflowing material collides with denser matter, causing strong shocks and giving rise to the observed $\mathrm{H}_{2}$ emission.

\subsubsection{A precessing outflow?}

Feature $\mathbf{F}$ is related to a much larger, strongly elongated structure pointing away from IRS 3 in a north-eastern direction that can be seen very well in the deep $K$-band image of the S140 IRS complex presented by Yao et al. (1998; their Fig. 2). In Yao's image, this structure can be clearly traced more than $15^{\prime \prime}$ away from IRS 3 with a position angle of $\sim 64^{\circ}$. Our feature $\mathbf{F}$ is the innermost and brightest part of this elongated nebulosity. The same elongated nebulosity can also be seen well in the deep $K$-band image of the S140 region presented by Hodapp (1994). Interestingly, this image also shows a diffuse patch of emission with a bow-shock like shape, located $90^{\prime \prime}$ north-east of IRS 3 at a position angle of $\sim 68^{\circ}$. It appears very likely that this bow-shock like patch is related to the elongated emission near IRS 3 and our feature $\mathbf{F}$.

Our result that feature $\mathbf{F}$ is associated with strong molecular hydrogen shock emission in combination with the presence of the bow-shock like feature further away strongly suggests that material is flowing away from IRS 3 in north-eastern direction. Our speckle image allows us to resolve the detailed morphology of the innermost part of this feature and reveals a remarkable wiggly S-shaped structure. This is best seen in the speckle image reconstructed with reduced resolution (Fig. 1 lower right). It is very tempting to speculate that this feature traces a precessing outflow originating from IRS $3 \mathrm{a}$. Therefore, we will now compare the general structure of this feature in our image with the jet precession model described by Eislöffel et al. (1996). In this model, the trajectory of a precessing jet as seen projected on the sky is given by the formula

$$
\begin{aligned}
f\left(\alpha, \lambda, \phi_{0}, \psi, l, x_{0}, y_{0}\right) & =\left(\begin{array}{cc}
\cos \psi & -\sin \psi \\
\sin \psi & \cos \psi
\end{array}\right) \\
& \times\left(\begin{array}{c}
\alpha l \sin \left(2 \pi l / \lambda+\phi_{0}\right) \\
l
\end{array}\right)+\left(\begin{array}{l}
x_{0} \\
y_{0}
\end{array}\right)
\end{aligned}
$$

where $\alpha$ is the precession amplitude, $\lambda$ the precession length, $\phi_{0}$ the initial phase at the source, $\psi$ the rotation angle of the flow in the plane of the sky, and $l$ the distance from the source position $x_{0}, y_{0}$. The model curve shown in Fig. 1 (lower right image) was obtained for values of $\alpha=0.25, \lambda=4.32^{\prime \prime}, \phi_{0}=190^{\circ}$, and $\psi=65^{\circ}$. This curve traces the observed morphology of feature $\mathbf{F}$ remarkably well. The parameters of the model curve shown in Fig. 1 yield a precession length scale of $\sim 4000$ AU. Assuming a flow velocity of $\sim 100-200 \mathrm{~km} \mathrm{~s}^{-1}$, as typically found for young stellar objects (cf. Eislöffel et al. 2000; Richer et al. 2000), the corresponding precession period would be $\sim 100-200$ yr.

If our interpretation is correct, the next question would be, what causes the precession of the outflow? One possible explanation might be that the outflow originates from a member of a close binary system where the rotational axis of the star driving the outflow is misaligned with the orbital plane of the binary. In their theoretical study of non-coplanar binary systems, Bate et al. (2000) found that the rotation axis of the star (and therefore also the outflow) precesses with a period of the order of 20 binary periods. In the case of IRS 3a, where we can assume a mass of $\sim 8 M_{\odot}$ (Lester et al. 1986), this model would require a binary with a separation of $\sim 6-9$ AU. At the 150 mas ( $\sim 135 \mathrm{AU})$ resolution of our image, we would clearly not be able to resolve such a close binary. Therefore, our observations are not inconsistent with the hypothesis of a precessing outflow originating from a non-coplanar binary system.

Finally, we note that our precessing outflow model is of course not the only possible explanation for the shape of feature $\mathbf{F}$. The observed $K$-band morphology might also be caused by projection effects when looking at a clumpy structure, or by inhomogeneous extinction. Another potential problem with our precessing outflow model might be the fact that we do not see any counterpart of this outflow, which would be expected at a position angle of $245^{\circ}$. However, this might simply be due to the inclination of the outflow axis with respect to the line-of-sight: if the $65^{\circ}$ outflow is not perpendicular to the line-of-sight but tilted in our direction, the expected counterpart would point away from us and therefore could well be hidden by circumstellar extinction.

\subsection{Diffuse emission south of IRS 3 a}

Features A, B, and $\mathbf{C}$ seem to point away from IRS 3a. One possible interpretation of these features may be that we see scattered light from the limb-brightened inner walls of a cavity in a circumstellar envelope around IRS 3a, similar to the extended emission we found south of S140 IRS 1 
(cf. Schertl et al. 2000). Another possibility would be that these features either represent outflowing material or that they trace shock-heated regions in which outflowing material collides with ambient medium. This second possibility is supported by the fact that we find strong $\mathrm{H}_{2}$ emission coinciding with feature $\mathbf{B}$, indicating the presence of shocked material. In this case, the quite large range of position angles of features $\mathbf{A}, \mathbf{B}$, and $\mathbf{C}\left(120^{\circ}\right.$ to $\left.\sim 180^{\circ}\right)$ might indicate a poorly collimated outflow.

Feature $\mathbf{C}$ displays a remarkable curved shape: it seems to start at IRS 3a in a southern direction and strongly turns to the east. From the origin to the tip of this feature its direction changes by nearly $90^{\circ}$. This remarkable curvature could be indicative of compression from the western direction. Interestingly, this is just the direction in which S140 IRS 1 is found, the most luminous source in the S140 IRS cluster. IRS 1 is expected to strongly affect its environment by several mechanisms. Firstly, IRS 1 is the source of strong outflow activity in several directions (cf. Weigelt et al. 2001). Secondly, from radio observations of the S140 IRS region, Evans et al. (1989) concluded that most of the radio emission from IRS 1 probably arises from an optically thin HII region, while some fraction originates from a jet or a collimated stellar wind. Both mechanisms, an expanding HII region as well as a strong stellar wind, can be expected to be of considerable influence on the ambient molecular cloud material. Therefore, it seems to be quite plausible that IRS 1 compresses the gas near IRS 3, distorting and bending the material as observed in feature $\mathbf{C}$.

In this context it is interesting to note that Bally \& Reipurth (2001) reported the discovery of several jets with strongly curved shapes. The authors offer two explanations for bent jets: the bending could be caused by sidewards compression, either by other outflows or by the irradiation from massive stars. Alternatively, the jet bending may indicate that the source star is ejected from their cluster. Masciadri \& Raga (2001) have recently presented three-dimensional gas-dynamical models of the interaction between outflowing material from a young stellar object and a wind perpendicular to the outflow direction. These models can reproduce the curved shapes of jets observed by Bally \& Reipurth (2001) very well and also show remarkable similarity to the morphology of feature $\mathbf{C}$.

\subsection{Features $\mathbf{D}$ and $\mathbf{E}$}

Our image also reveals two compact features, D and E. Despite their point-source like appearance, we find that these two features are clearly more extended than expected for a point source. Feature $\mathbf{D}$ could be connected to IRS $3 b$, but we cannot exclude a chance projection. Feature $\mathbf{E}$ appears to be an isolated blob and shows strong $\mathrm{H}_{2}$ emission. This suggests that it may be related to the outflow system south of IRS 3a. Proper motion measurements of these features, interpreted as ballistic motions, could soon determine if these features are associated with outflows from any of the infrared sources.

\section{On the IRS 3 triple system}

Our images show that IRS 3 consists of (at least) three point sources. Of course, with the data at hand we cannot be sure that these three point sources are physically related and actually constitute a triple system; they might perhaps just be a chance projection. However, the fact that our image does not show any other point sources in the full $10.8^{\prime \prime} \times 10.8^{\prime \prime}$ field-of-view allows us to, at least roughly, evaluate the likelihood of a chance projection. If the three point sources were unrelated objects, the probability to find all three within $1.3^{\prime \prime}$ of each other would be $P=\left(\frac{\pi\left(1.3^{\prime \prime}\right)^{2}}{\left(10.8^{\prime \prime}\right)^{2}}\right)^{2}=0.00207$. It is therefore reasonable to assume that the three sources are related and thus constitute a triple system.

It is interesting to make an assessment of how stable the system is. A rather general result about triple systems is that they can be stable only if they are sufficiently hierarchical. Eggleton \& Kiseleva (1995) derived the following relation between the minimum initial ratio $Y_{0}^{\text {min }}$ of the periastron distance of the outer orbit to the apastron distance of the inner orbit for a stable triple system:

$Y_{0}^{\mathrm{min}} \approx 1+\frac{3.7}{q_{\text {out }}^{1 / 3}}+\frac{2.2}{1+q_{\text {out }}^{1 / 3}}+\frac{1.4}{q_{\text {in }}^{1 / 3}} \frac{q_{\text {out }}^{1 / 3}-1}{q_{\text {out }}^{1 / 3}+1}$

where $q_{\text {in }}=M_{\mathrm{a}} / M_{\mathrm{b}}$ and $q_{\text {out }}=\left(M_{\mathrm{a}}+M_{\mathrm{b}}\right) / M_{\mathrm{c}}$ and $M$ denotes the stellar masses of the individual components.

Lacking detailed information about the orbits of the IRS 3 components, we assume that the system is coplanar and the orbits are circular. Using the projected separations of the components in our image as estimates for their orbital radii, we then find $Y_{0} \approx \rho_{\mathrm{ac}} / \rho_{\mathrm{ab}}=1306 / 627 \approx 2.1$.

Estimates for the masses of the IRS 3 components can be derived in the following way: first, we assume a mass of $M_{\mathrm{a}} \sim 8 M_{\odot}$ for the primary component IRS 3a based on the estimate of its luminosity (Lester et al. 1986). From this, we can then estimate the masses of the other two components from their $K$-band magnitude differences and find: $M_{\mathrm{b}} \sim 5 M_{\odot}$, and $M_{\mathrm{c}} \sim 3-4 M_{\odot}$. Based on these mass estimates (and the above estimate of the orbital radii), we plotted the parameters of IRS 3 in Fig. 2 and find that, even allowing generously for large uncertainties in the system parameters, the IRS 3 triple system appears to be unstable.

In a recent paper, Reipurth (2001) postulated that the dynamical decay of triple or higher order multiple systems can lead to strong outflow activity: as the system breaks up and ejects the lightest member, massive disk truncation and accompanying large-scale accretion in one of the remaining stars causes a burst of outflow activity. This model could provide a good explanation for the IRS 3 system: IRS 3c, the faintest and therefore probably lightest component of the system, is being ejected, whereas IRS $3 \mathrm{a}$ is undergoing a phase of strong outflow activity. 


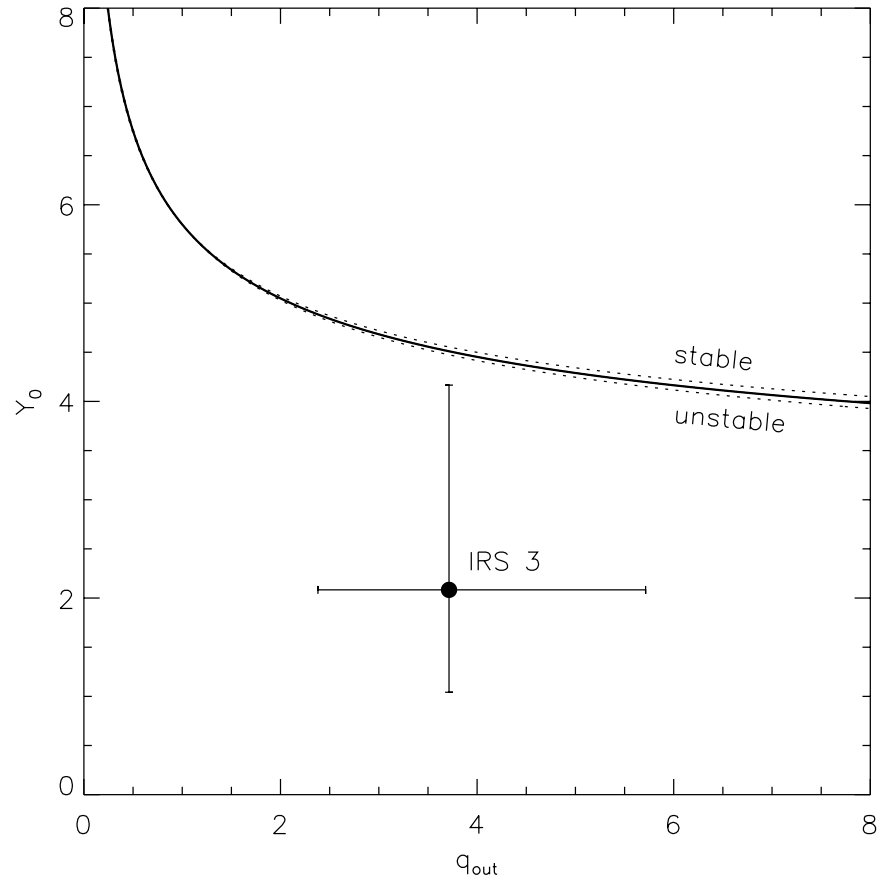

Fig. 2. The stability criterion for triple systems (Eq. (2)) is shown as the thick solid line. The thin dotted lines indicate the range of variations resulting from the uncertainty of $q_{\text {in }}$. Systems above the boundary are stable, systems below the boundary are unstable. Our estimate of the parameters for the IRS 3 system is shown as the solid dot with error bars. We assume a factor of 2 uncertainty for $Y_{0} \approx \rho_{\text {ac }} / \rho_{\text {ab }}$, and $20 \%$ uncertainties for the mass estimates.

We note that it is unclear how this scenario would be affected if IRS 3a actually is a close binary, as we proposed in the last section to explain the precession of the outflow. In this case, we would deal with a quadruple system, and the kinematic would be much more complicated. Nevertheless, this scenario would probably still work.

Another interesting aspect to consider in this context is the corresponding timescale. If one of the stars is actually ejected from the system, then the rather compact configuration of the system, with stellar separations not more than $\sim 1200 \mathrm{AU}$, suggests that the kinematic interaction causing the ejection took place not long ago, probably not more than a few thousand years ago. This would imply that the outflow activity also only started very recently. This might explain why, so far, no large-scale CO outflow has been detected from IRS 3: the outflow from IRS 3 is probably so young that it has not yet swept up enough material to be detectable in the molecular maps.

In future observations we hope to be able to determine the proper motions of the individual objects in IRS 3. This will provide us with important information on the dynamics of the IRS 3 system and show whether the ejection scenario is applicable to this system.

\section{Conclusions}

The main results of our high-resolution near-infrared imaging study of S140 IRS 3 can be summarized as follows:

1. S140 IRS 3 is resolved as a triple system with separations of $0.63^{\prime \prime}(\sim 560 \mathrm{AU})$ and $1.3^{\prime \prime}(\sim 1200 \mathrm{AU})$. A rough evaluation of the system parameters suggests that the IRS 3 triple system is most likely unstable.

2. Our image reveals a remarkable S-shaped diffuse emission feature north-east of IRS 3a, which is the innermost part of a strongly elongated extended feature pointing towards a bow-shock $90^{\prime \prime}$ north-east of IRS 3 a. We find this feature to be associated with strong $\mathrm{H}_{2}$ line emission. Its morphology can be well explained with a model for a precessing outflow. The precession could be caused by a close binary system in which the axis of the outflow source is mis-aligned with the orbital plane.

3. Some parts of the bright diffuse emission to the south of IRS 3a are associated with strong $\mathrm{H}_{2}$ line emission and therefore seem to trace outflowing material. One of these features is strongly curved; this could represent a bended jet or outflow. The bending may be explained as due to compression of the material in this region from the west, caused by the strong outflow source IRS 1.

Acknowledgements. We would like to thank the Calar Alto staff for their support during our observations, Thomas Stanke for assistance in the reduction of the Omega data, and the referee T. Nagata for his report which helped to improve this paper.

\section{References}

Bally, J., \& Reipurth, B. 2001, ApJ, 546, 299

Bate, M. R., Bonnell, I. A., Clarke, C. J., et al. 2000, MNRAS, 317,773

Beichman, C. A., Becklin, E. E., \& Wynn-Williams, C. G. 1979, ApJ, 232, L47

Bizenberger, P., McCaughrean, M. J., Birk, C., Thompson, D., \& Storz, C. 1998, Proc. SPIE, 3354, 825

Blair, G. N., Evans, N. J. II, Vandenbout, P. A., \& Petters, W. L. III 1978, ApJ, 219, 896

Crampton, D., \& Fisher, W. A. 1974, Pub. Dom. Ap. Obs., 14, 12

Eggleton, P., \& Kiseleva, L. 1995, ApJ, 455, 640

Eislöffel, J., Smith, M. D., Davis, C. J., \& Ray, T. P. 1996, AJ, 112,2086

Eislöffel, J., Mundt, R., Ray, T. P., \& Rodriguez, L. F. 2000, in Protostars and Planets IV, ed. V. Mannings, A. P. Boss, \& S. S. Russel (Tucson: University of Arizona Press), 815

Evans, N. J. II, Mundy, L. G., Kutner, M. L., \& DePoy, D. L. 1989, ApJ, 346, 212

Harker, D., Bregman, J., Tielens, A. G. G. M., Temi, P., \& Rank, D. 1997, A\&A, 324, 629

Hayashi, M., \& Murata, Y. 1992, PASJ, 44, 391

Hofmann, K.-H., \& Weigelt, G. 1986, A\&A, 167, L15

Hodapp, K.-W. 1994, ApJS, 94, 615 
Labeyrie, A. 1970, A\&A, 6, 85

Lester, D. F., Harvey, P. M., Joy, M., \& Ellis, H. B. Jr. 1986, ApJ, 309, 80

Lohmann, A. W., Weigelt, G., \& Wirnitzer, B. 1983, Appl. Opt., 22, 4028

Masciadri, E., \& Raga, A. C. 2001, AJ, 121, 408

McCaughrean, M. J., Zinnecker, H., \& Rayner, J. T. 1994, ApJ, 436, L189

Minchin, N. R., White, G. J., \& Padman, R. 1993, A\&A, 277, 595

Reipurth, B. 2001, ApJ, in press

Richer, J., Shepherd, D., Cabrit, S., Bachiller, R., \& Churchwell, E. 2000, in Protostars and Planets IV, ed. V. Mannings, A. P. Boss, \& S. S. Russel (Tucson: University of Arizona Press), 867
Rouan, D., Lena, P. J., Puget, J. L., de Boer, K. S., \& Wijnbergen J. J. 1977, ApJ, 206, L35

Schertl, D., Balega, Y., Hannemann, T., et al. 2000, A\&A, 361, L29

Smith, M. D. 1993, ApJ, 406, 520

Weigelt, G. 1977, Opt Commun., 21, 55

Weigelt, G. 1991, in Progress in Optics, vol. 29, ed. E. Wolf (Elsevier Science Publishers), 295

Weigelt, G., Preibisch, Th., Schertl, D., Balega, Y. Y., \& Smith, M. D. 2001, A\&A, submitted

Wilner, D. J., \& Welch, W. J. 1994, ApJ, 427, 898

Whitney, B. A., Kenyon, S. J., \& Gomez, M. 1997, ApJ, 485, 703

Yao, Y., Ishi, M., Nagata, T., et al. 1998, ApJ, 500, 320 